\title{
Ischemic postconditioning protects the spinal cord from ischemia-reperfusion injury via modulation of redox signaling
}

\author{
Wenying Song, MD, , ${ }^{\text {ab }}$ Jing Sun, MD, $\mathrm{PhD},{ }^{\text {a }}$ Binxiao $\mathrm{Su}, \mathrm{MD}, \mathrm{PhD},{ }^{\mathrm{a}}$ Rui Yang, MD, \\ Hailong Dong, $\mathrm{MD}, \mathrm{PhD},{ }^{\mathrm{a}}$ and Lize Xiong, $\mathrm{MD}, \mathrm{PhD}^{\mathrm{a}}$
}

\begin{abstract}
Background: It is well known that ischemic postconditioning reduces ischemic-reperfusion injury, but the underlying mechanism is not fully understood. The current study investigated the role of reactive oxygen species-mediated upregulation of endogenous antioxidant enzymes in the generation of a protective effect induced by ischemic postconditioning against spinal cord reperfusion injury in the rabbit.
\end{abstract}

\begin{abstract}
Methods: New Zealand White rabbits were randomly allocated to sham, ischemia-reperfusion, and postconditioning groups ( 3 cycles of 30 seconds of reperfusion and 30 seconds of occlusion during the onset of reperfusion). Spinal cord ischemia was induced by clamping the infrarenal abdominal aorta for 20 minutes in the ischemia-reperfusion and postconditioning groups. Forty-eight hours after reperfusion, the neurologic status of the lower limbs was assessed. Blood samples were collected for analysis of serum neuron-specific enolase levels, and the lumbar spinal cord segments (L5-7) were harvested for histopathologic and antioxidant enzyme activities and mRNA analysis with or without administration of N-2-mercaptopropionylglycine (an effective oxygen free radical scavenger) given at different reperfusion times.
\end{abstract}

Results: Continuous administration of N-2-mercaptopropionylglycine for 13 minutes, starting at 10 minutes before (but not 10 minutes after) the beginning of reperfusion, attenuated the neuroprotective effect of postconditioning against spinal cord ischemia and reversed the increase in activity of the antioxidant enzymes superoxide dismutase and catalase in spinal cord tissue subjected to ischemic postconditioning.

Conclusions: The results indicate that reactive oxygen species-triggered upregulation of endogenous antioxidant enzyme activities may be involved in the mechanism of neuroprotection of ischemic postconditioning. (J Thorac Cardiovasc Surg 2013;146:688-95)

Despite countless therapeutic strategies and clinical studies to address ischemia-reperfusion (I/R) injury, paraplegia related to the surgical repair of descending and thoracoabdominal aortic aneurysms still occurs. ${ }^{1,2}$ Therefore, novel measures to protect the spinal cord against surgical ischemic injury require further exploration.

After the concept of attenuating reperfusion injury was introduced, ${ }^{3}$ ischemic preconditioning (IPC) was first proposed by Murry and colleagues ${ }^{4}$ in 1986 . IPC had a powerful endogenous protection to enhance the tolerance against

From the Department of Anesthesiology, ${ }^{a}$ Xijing Hospital, Fourth Military Medical University, Xi' an, Shaanxi Province, China; and Department of Anesthesiology, ${ }^{\text {b }}$ Shaanxi Province People's Hospital, Xi'an, Shaanxi Province, China.

Supported by the National Natural Science Foundation of China (Beijing, China, Grants 30772059, 30972853, and 81128005 to H.D., and Grant 81100901 to B.S.), the National Science Foundation for Distinguished Young Scholars (Beijing, China, Grant 30725039 to L.X.), and the Major Program of National Natural Science Foundation of China (Beijing, China, Grant 30930091 to L.X.).

Disclosures: Authors have nothing to disclose with regard to commercial support.

W.S. and J.S. are co-first authors.

Received for publication July 25, 2012; revisions received Oct 31, 2012; accepted for publication Nov 12, 2012; available ahead of print Dec 14, 2012.

Address for reprints: Lize Xiong, MD, PhD, and Hailong Dong, MD, PhD, Department of Anesthesiology, Xijing Hospital, Fourth Military Medical University, Xi'an, Shaanxi Province, P. R. China (E-mail: mzkxlz@126.com and donghl@ fmmu.edu.cn).

$0022-5223 / \$ 36.00$

Copyright (c) 2013 by The American Association for Thoracic Surgery

http://dx.doi.org/10.1016/j.jtcvs.2012.11.039 ischemia in many organs, such as the heart, brain, and spinal cord. Because the onset of ischemia cannot be predicted and IPC implementation may present a potential risk to patients, IPC is unfeasible in clinical situations. A novel approach termed "postconditioning" (PostC), in which brief repeated cycles of intermittent reperfusion and reocclusion are applied at the onset of reperfusion after a prolonged period of ischemia, significantly reduced myocardial infarct size. ${ }^{5}$ PostC has been successfully used in clinical procedures. ${ }^{6}$ Our preliminary study demonstrated that PostC, consisting of 3 cycles of 30 seconds of reperfusion and 30 seconds of reocclusion, significantly preserved locomotor function and decreased the paraplegia rate in rabbits subjected to 20 minutes of spinal cord ischemia. Activities of superoxide dismutase (SOD) and catalase in the spinal cord tissue of animals in the PostC group were significantly higher compared with the control group at approximately 30 minutes and 6 hours after reperfusion. ${ }^{7}$ However, whether the increase of antioxidant enzyme activities is directly associated with the mechanism of neuroprotection of PostC against reperfusion injury remains unknown.

After an ischemic stroke, the production of reactive oxygen species (ROS) may increase, leading to tissue damage. ${ }^{8}$ However, ROS have been paradoxically implicated as an essential signaling component in protective pathways that 


\section{Abbreviations and Acronyms \\ GAPDH $=$ glyceraldehyde 3-phosphate dehydrogenase \\ IPC $=$ ischemic preconditioning \\ $\mathrm{I} / \mathrm{R}=$ ischemia-reperfusion \\ MPG = N-2-mercaptopropionylglycine \\ NSE $=$ neuron-specific enolase \\ PostC = postconditioning \\ ROS $=$ reactive oxygen species \\ SOD $=$ superoxide dismutase}

mediate the effect of IPC ${ }^{9}$ and pharmacologic PostC. ${ }^{10}$ ROS are obligatory mediators of the signal transduction pathway to upregulate antioxidant enzymes and induce the cardiac protective effects of ischemic PostC. ${ }^{11}$ Oxidative stress related to the enormous generation of ROS may play an important part in reperfusion injury. Excessive ROS production is considered injurious to cell function and mitochondria. On the other hand, low levels of ROS may perform as secondary messengers, modulating signaling pathways by covalent medications of target molecules, which is referred to as "redox signaling." The current study attempts to provide evidence for the role of ROS in the mechanism of ischemic PostC in a rabbit spinal cord ischemic model.

\section{MATERIALS AND METHODS}

All experiments performed in this study were approved by the Ethics Committee for Animal Experimentation and conducted according to the Guidelines for Animal Experimentation of the Fourth Military Medical University. The study was conducted in Xijing Hospital, Fourth Military Medical University (Xi'an, China).

\section{Animal Care}

Male New Zealand White rabbits weighing 2.2 to $2.5 \mathrm{~kg}$ were obtained from the experimental Animal Center of the Fourth Military Medical University, Xi' an, China, and were housed in an animal center in Xijing Hospital. The experiments described in this study were approved by the Committee of Animal Study of the Fourth Military Medical University. The animals had access to standard rabbit chow and water ad libitum.

\section{Experimental Protocol}

Part I of the experiment determined the effect of N-2mercaptopropionylglycine (MPG) on the induction of ischemic tolerance and antioxidant enzyme activities by PostC. To detect the effect of MPG on the neuroprotection of PostC against spinal cord I/R injury, 84 male New Zealand White rabbits were randomly assigned to 8 groups $(n=6$ in each group; Figure 1). Except for the sham group, all animals were subjected to occlusion of the infrarenal abdominal aorta for 20 minutes, followed by reperfusion for 48 hours with or without PostC ( 3 cycles of 30 seconds of reperfusion and 30 seconds of ischemia). Animals in the vehicle group received an intraperitoneal injection of deionized water, and animals in the MPG and PostC + MPG groups received an intravenous injection of MPG dissolved in deionized water (Sigma-Aldrich, St Louis, Mo), a potent ROS scavenger, at $0.5 \mathrm{~mL} \cdot \mathrm{kg}^{-1} \cdot \mathrm{min}^{-1}$ with a micro-syringe pump for 13 minutes (started 10 minutes before reperfusion). In the MPG(D) and PostC + MPG(D) groups, MPG was administered 10 minutes after reperfusion for 13 minutes. The dose and administration method of MPG in this experiment were chosen in line with previous research. ${ }^{12}$ At 48 hours after reperfusion, the blood samples were collected for analysis of serum neuron-specific enolase (NSE) levels, the motor function of the hind limbs was scored, and the number of normal motor neurons at the anterior horn of the spinal cord (L5) was counted in a blinded fashion.

Part II of the experiment investigated the effect of MPG on antioxidant enzyme activities (SOD and catalase) after PostC. Thirty male rabbits were randomly divided into 5 groups ( $\mathrm{n}=6$ for each group). All animals were subjected to occlusion of the infrarenal abdominal aorta for $20 \mathrm{~min}$ utes, followed by reperfusion for 6 hours. Animals in the Con and PostC groups received a deionized water injection, and animals in the MPG and PostC + MPG groups received MPG $\left(2 \%[\mathrm{w} / \mathrm{v}], 0.5 \mathrm{~mL} \cdot \mathrm{kg}^{-1} \cdot \mathrm{min}^{-1}\right)$ 10 minutes before reperfusion. After the neurologic outcomes were evaluated 6 hours after reperfusion, the spinal cord tissue (L5-7) was harvested and frozen at $-80^{\circ} \mathrm{C}$ to determine SOD and catalase activities by spectrophotometric methods as previously described. ${ }^{7,9}$ mRNA expression levels of the antioxidant enzymes were measured by semiquantitative reverse transcriptase polymerase chain reaction.

\section{Surgical Preparation}

Animals were neurologically intact before anesthesia and instrumentation. General anesthesia was induced by intravenous injection of sodium pentobarbital $(30 \mathrm{mg} / \mathrm{kg}){ }^{9}$ Rabbits did not receive mechanical ventilation support throughout the procedure and were allowed to breathe spontaneously with an oxygen facemask at a flow rate of $2 \mathrm{~L} / \mathrm{min}$. Lactated Ringer's solution was infused intravenously at a rate of $4 \mathrm{~mL} \cdot \mathrm{kg}^{-1} \cdot \mathrm{h}^{-1}$. In all animals, a 22-gauge catheter was inserted into the ear artery to measure the proximal blood pressure and sample arterial blood. Another catheter was inserted into the left femoral artery to measure femoral blood pressure. Arterial blood pressure and heart rate were monitored continuously using a calibrated pressure transducer connected to an invasive pressure monitor (Colin BP-508 type S; Omron Colin, Tokyo, Japan). Rectal temperature was maintained at $38.5^{\circ} \mathrm{C} \pm 0.5^{\circ} \mathrm{C}$ using a heating blanket and overhead lamp. Arterial blood was sampled at baseline, preischemia, 10 minutes after ischemia, the end of PostC, and 10 minutes after reperfusion to determine the arterial oxygen tension, arterial carbon dioxide tension, $\mathrm{pH}$, and blood glucose. Arterial blood gases were measured using the Rapid Lab 1260 (Bayer HealthCare AG, Leverkusen, Germany).

\section{Spinal Cord Ischemia}

Spinal cord ischemia was induced by infrarenal aortic occlusion as described in our previous studies. ${ }^{9,13}$ Briefly, the rabbits were placed in the supine position after local anesthesia. The abdominal aorta was exposed at the level of the left renal artery through a 3- to 4-cm-long medial incision. Heparin (400 units) was administered 5 minutes before aortic occlusion. Spinal cord ischemia was induced by clamping the aorta with a bulldog clamp just below the renal artery. Obstruction of blood flow lasted 20 minutes. The bulldog clamp was removed, and stabilization of arterial blood pressure was monitored for an additional 20 minutes. The abdominal wall was closed with wound clips. Local infiltration with $0.25 \%(\mathrm{w} / \mathrm{v}) \mathrm{bu}-$ pivacaine hydrochloride around the wound was applied for postoperative analgesia. An antibiotic (40,000 units of gentamicin) was administered intramuscularly immediately after the operation. Animals were placed on a heating pad to maintain body temperature until recovery from anesthesia and then were returned to their home cage. Animals survived for 2 days. Bladder content was compressed manually as required.

\section{Neurologic Outcome Assessment}

Neurologic status was assessed 6, 24, and 48 hours after reperfusion by a member of the research team who was unaware of the grouping. Modified Tarlov criteria were used: $0=$ no voluntary hind-limb function, $1=$ only perceptible joint movement, 2 = active movement but unable 


\begin{tabular}{|c|c|c|}
\hline Sham & $20 \mathrm{~min}$ & $48 \mathrm{hR}$ \\
\hline \multirow[t]{2}{*}{ Con } & $20 \mathrm{~min} I$ & $48 \mathrm{~h} \mathrm{R}$ \\
\hline & 0.55 & ore $\mathrm{R}$ \\
\hline \multirow[t]{2}{*}{ MPG } & $20 \min I$ & $48 \mathrm{~h} \mathrm{R}$ \\
\hline & & $1,1, \mathrm{MPG}, \mathrm{iN}, 10 \mathrm{~min}$ after $\mathrm{R}$ \\
\hline MPG(D) & $20 \min I$ & $48 \mathrm{~h} \mathrm{R}$ \\
\hline \multirow[t]{2}{*}{ Postc } & $20 \mathrm{~min} I$ & $48 \mathrm{~h} \mathrm{R}$ \\
\hline & & fore $\mathrm{R}$ \\
\hline \multirow[t]{2}{*}{ PostC+MPG } & $20 \mathrm{~min} \mathrm{I}$ & $48 \mathrm{~h} \mathrm{R}$ \\
\hline & & In-1,MPG, iv, 10 min after PostC \\
\hline \multirow[t]{2}{*}{ PostC+MPG(D) } & $20 \min$ & $48 \mathrm{~h} \mathrm{R}$ \\
\hline & & 0 min before $R$ \\
\hline Vehicle & $20 \mathrm{~min} I$ & $48 \mathrm{~h} \mathrm{R}$ \\
\hline
\end{tabular}

FIGURE 1. Time lines and protocols for part 1 of the experiment $(n=6$ for each group). All rabbits underwent 20 minutes of spinal cord ischemia (occlusion of the infrarenal abdominal aorta) and 48 hours of reperfusion, except the sham group. Con: I/R group. MPG: Rabbits were treated with the potent ROS scavenger MPG 10 minutes before reperfusion for 13 minutes. MPG(D): Rabbits were treated with delayed MPG, 10 minutes after reperfusion, for 13 minutes. Ischemic PostC: Rabbits received 3 cycles of 30 seconds of reperfusion and 30 seconds of occlusion during the onset of reperfusion. PostC + MPG: Rabbits were treated with MPG before and during PostC (10 minutes before reperfusion for 13 minutes). PostC + MPG(D): Rabbits were treated with delayed MPG after PostC, 10 minutes after reperfusion, for 13 minutes. $R$, Reperfusion; $M P G, \mathrm{~N}-2-$ mercaptopropionylglycine; PostC, postconditioning.

to stand, $3=$ able to stand but unable to walk, and $4=$ complete normal hind-limb motor function. ${ }^{9,14,15}$

\section{Hematoxylin-Eosin Staining and Cell Counting}

In part I of the experiment, after neurologic testing at 48 hours after reperfusion, the rabbits were deeply anesthetized and transcardially perfused/ fixed with heparinized saline followed by $10 \%(\mathrm{v} / \mathrm{v})$ buffered formalin. After perfusion, L5-7 segments of the spinal cord were dissected and removed. The spinal cord blocks were postfixed for 48 hours in the same fixatives and embedded in paraffin. Coronal sections $(4 \mu \mathrm{m})$ of the L5 segment were stained with hematoxylin-eosin.

A neuropathologist who was blinded to the group and experimental procedures performed the histologic evaluation using a light microscope (Olympus BX51 reflected system, digital camera DP 70, and visual communication suite Olympus DP-control; Olympus Corp, Tokyo, Japan) to determine the motor neurons in the anterior spinal cord (anterior to an imaginary line drawn through the central canal perpendicular to the vertical axis). Averages of the number of normal neurons in the anterior horn in the unilateral side were compared among groups. The remaining normal neurons were distinguished on the basis of cell morphology (a clear nucleus and prominent nucleolus), whereas injured neurons were identified by an intense eosinophilic cytoplasm with loss of Nissl substance and by the presence of pyknotic homogenous nuclei. The number of normal motor neurons in 5 sections, selected alternatively, was counted in each animal and averaged.

\section{Neuron-Specific Enolase Analysis}

In part I, blood samples were collected and centrifuged (5000 rpm, 10 minutes at $\left.4^{\circ} \mathrm{C}\right) 48$ hours after reperfusion and kept frozen at $-80^{\circ} \mathrm{C}$ until studied. NSE was measured using chemiluminescent immunometric assays (Roche Applied Science, Indianapolis, Ind; Catalogue no. 12133121122), and the results were expressed as nanograms/milliliter.

\section{Measurement of Antioxidant Enzyme Activity}

In part II of the experiment, animals were killed 6 hours after reperfusion, and the spinal cords (L5-7) were quickly harvested, weighed, and homogenized. All procedures were performed at $0^{\circ} \mathrm{C}$. The homogenate was centrifuged at $3000 \mathrm{r} / \mathrm{g}$ for 15 minutes at $4^{\circ} \mathrm{C}$, and the supernatant was collected and frozen at $-80^{\circ} \mathrm{C}$ for the next assay. Measurements of SOD and catalase activities in tissue homogenates were processed spectrophotometrically according to the specifications of the detection kit provided by the manufacturer (Jian Chen Biologic Institute, Nanjing, Jiangsu, China). ${ }^{9}, 16,17$

Xanthine-xanthine oxidase was used to generate superoxide flux. The absorbance obtained from the reduction of nitroblue tetrazolium to a blue formazan product by superoxide was determined at $560 \mathrm{~nm}$ spectrophotometrically at room temperature. One unit of SOD activity is defined as the amount that reduces the absorbance change by $50 \%$, and the activity was normalized on the basis of total protein content (units/ milligram $\cdot$ protein).

Catalase activity in spinal cord tissue was determined by spectrophotometric methods based on the hydrogen peroxide decomposition rate at 240 $\mathrm{nm}$ (using an ultraviolet light spectrophotometer, Beckman Coulter Inc, Fullerton, Calif) in the reactive mixture. Catalase activity was given in units/milligram protein.

\section{Reverse Transcriptase Polymerase Chain Reaction}

The total RNA was extracted from spinal cord tissues by TRIzol (Invitrogen Technology, Carlsbad, Calif) according to the manufacturer's directions. Gene-specific primers for $\mathrm{Cu}, \mathrm{Zn}$-SOD (forward: $5^{\prime}$-GTGTGCGTG CTGAAGGGCGA- $3^{\prime}$ and reverse: 5'-CATTTCCACCTTTGCCCAAG TC- $3^{\prime}$ ), catalase (forward: $5^{\prime}$ - AGGGTGGTGCTCCCAACTACTAC- $3^{\prime}$ and reverse: $5^{\prime}$ - GGCTTCTGGGAGTTGTACTGGTC- $3^{\prime}$ ), and glyceraldehyde 3-phosphate dehydrogenase (GAPDH) (forward: $5^{\prime}$ - forward: 5'- TGGTCACCAGGGCTGCTTTTAACT-3' and reverse: 5'-GCTAAG CAGTTGGTGGTGCAGGA- $3^{\prime}$ ) were adopted from the study by Ciqremis and colleagues. ${ }^{18}$ Equal amounts of RNA (500 ng) from each sample were reverse transcribed in a volume of $10 \mu \mathrm{L}$ to produce cDNA using the Takara RNA PCR Kit (AMV) version 3.0 (TaKaRa Bio Inc, Otsu, Japan). The reaction conditions were as follows: initial denaturation at $94^{\circ} \mathrm{C}$ for 3 minutes, denaturation at $94^{\circ} \mathrm{C}$ for 30 seconds, annealing at $60^{\circ} \mathrm{C}(\mathrm{Cu}, \mathrm{Zn}-\mathrm{SOD}$ and $\mathrm{GAPDH}$ ) or $45^{\circ} \mathrm{C}$ (catalase) for 30 seconds, and extension at $72^{\circ} \mathrm{C}$ for 30 seconds. There were 32 polymerase chain reaction cycles. Standard curves plotting the threshold amplification cycle number values against input quantity for each gene were constructed using 5-fold serial dilutions of the reverse transcriptase product. GAPDH served as an endogenous internal standard control. Detection based on SYBR Green I (Applied Biosystems, Foster City, Calif) was carried out on a real-time polymerase chain reaction instrument (ABI PRISM 7300; Applied Biosystems) with a thermal cycler.

\section{Statistical Analysis}

All data, excluding the neurologic function score, are expressed as the mean \pm standard error of the mean. ${ }^{19}$ The neurologic function scores are expressed as the median (minimum-maximum value). The physiologic variables, serum NSE level, antioxidant enzyme activities, and mRNA in the spinal cord were analyzed using 1-way analysis of variance followed by the Student-Newman-Keuls test for multiple comparisons. The scores of hind-limb motor function and the number of normal neurons in the anterior spinal cord were analyzed with the nonparametric Kruskal-Wallis test followed by the Mann-Whitney $U$ test with Bonferroni correction. All statistical analyses were performed using the Statistical Package for the Social Sciences version 13.0 (SPSS Inc, Chicago, Ill). 


\section{RESULTS}

\section{Physiologic Variables}

There were no differences in relevant physiologic parameters among the groups regardless of treatment patterns. The value of distal blood pressure decreased to 7 to 11 $\mathrm{mm} \mathrm{Hg}$ after the abdominal artery was clamped. Ten minutes after the beginning of reperfusion, the value was restored to approximately preischemic levels (Table 1). During the experiment, the rectal temperature was maintained between $38^{\circ} \mathrm{C}$ and $39^{\circ} \mathrm{C}$ by an overhead lamp.

\section{Neurobehavioral Manifestation}

All animals survived until the final neurologic evaluation at 48 hours after reperfusion. The neurologic function scores after reperfusion are shown in Figure 2, $C$. All animals in the Con group developed paraplegia in the hind limb (Tarlov score $\leq 2) 48$ hours after reperfusion, whereas 4 animals in the PostC group had preserved motor function (Tarlov score $\geq 3$ ). The PostC group showed significantly higher hind-limb motor function scores than the Con group ( $P=.002$ vs the Con group). Nevertheless, the neurologic function outcome in the PostC + MPG group was worse than in the PostC group 48 hours after reperfusion $(P=.004$ PostC vs PostC + MPG) . There were no significant differences between the PostC and the PostC + MPG(D) groups $(P=.699)$.

\section{Histopathologic Outcomes}

The histopathologic results at 48 hours after reperfusion are shown in Figure 2, $A$ and $B$. Compared with the Con group, there were more normal neurons observed in the anterior spinal cord of the animals in the PostC group $(P<.001$ vs Con $)$ and PostC + MPG(D) group $(P<.001$ vs Con). However, there was no significant difference in the numbers of normal neurons between the Con group and the PostC + MPG group $(P=.382)$.

\section{Serum Neuron-Specific Enolase Levels}

There was a significant increase in serum NSE levels in the control animals compared with the sham animals. The NSE levels obtained in the PostC $(P=.026$ vs Con; Figure 3) and PostC + MPG(D) $(P=.036$ vs Con; Figure 3) groups were significantly lower than in the Con group, and there was no significant difference compared with the sham group. There was no difference in serum NSE levels between the PostC and PostC + MPG(D) groups $(P=.885)$.

\section{Antioxidant Activities}

As in Part 1, the rabbits in the PostC group had significantly higher hind-limb motor function scores than the rabbits in the Con group 6 hours after reperfusion $(P=.04$; Figure $4, A)$. A higher activity of SOD $(P=.009$ vs Con $)$

TABLE 1. Physiologic parameters for experiment $1(n=6)$

\begin{tabular}{|c|c|c|c|c|c|c|c|c|}
\hline & \multicolumn{2}{|c|}{ MAP (mm Hg) } & \multirow[b]{2}{*}{ HR (beats/min) } & \multirow[b]{2}{*}{$\mathbf{T}\left({ }^{\circ} \mathbf{C}\right)$} & \multirow[b]{2}{*}{ BG (mmol/L) } & \multirow[b]{2}{*}{ pH } & \multirow[b]{2}{*}{$\mathrm{PaO}_{2}(\mathrm{~mm} \mathrm{Hg})$} & \multirow[b]{2}{*}{$\mathrm{PaCO}_{2}(\mathrm{~mm} \mathrm{Hg})$} \\
\hline & Proximal & Distal & & & & & & \\
\hline \multicolumn{9}{|l|}{ Preischemia } \\
\hline $\mathrm{I} / \mathrm{R}$ & $89 \pm 1$ & $91 \pm 1$ & $261 \pm 5$ & $38.6 \pm 0.1$ & $6.2 \pm 0.1$ & $7.37 \pm 0.02$ & $102 \pm 1$ & $37 \pm 1$ \\
\hline PostC & $90 \pm 1$ & $91 \pm 2$ & $259 \pm 5$ & $38.6 \pm 0.1$ & $6.0 \pm 0.2$ & $7.36 \pm 0.01$ & $103 \pm 1$ & $36 \pm 0$ \\
\hline $\mathrm{I} / \mathrm{R}+\mathrm{MPG}$ & $91 \pm 1$ & $90 \pm 2$ & $257 \pm 5$ & $38.6 \pm 0.0$ & $6.2 \pm 0.2$ & $7.37 \pm 0.03$ & $101 \pm 1$ & $37 \pm 1$ \\
\hline PostC + MPG & $89 \pm 1$ & $90 \pm 2$ & $252 \pm 5$ & $38.5 \pm 0.1$ & $6.1 \pm 0.1$ & $7.38 \pm 0.04$ & $97 \pm 2$ & $37 \pm 1$ \\
\hline $\mathrm{I} / \mathrm{R}+(\mathrm{D}) \mathrm{MPG}$ & $88 \pm 2$ & $89 \pm 2$ & $249 \pm 5$ & $38.5 \pm 0.1$ & $6.2 \pm 0.2$ & $7.39 \pm 0.04$ & $96 \pm 1$ & $36 \pm 1$ \\
\hline PostC + (D)MPG & $91 \pm 1$ & $91 \pm 1$ & $256 \pm 4$ & $38.6 \pm 0.1$ & $6.1 \pm 0.1$ & $7.36 \pm 0.02$ & $103 \pm 1$ & $36 \pm 1$ \\
\hline \multicolumn{9}{|l|}{ Ischemia $10 \mathrm{~min}$} \\
\hline $\mathrm{I} / \mathrm{R}$ & $86 \pm 1$ & $9 \pm 0$ & $261 \pm 5$ & $38.4 \pm 0.2$ & $6.5 \pm 0.2$ & $7.38 \pm 0.03$ & $103 \pm 1$ & $34 \pm 1$ \\
\hline PostC & $89 \pm 1$ & $10 \pm 0$ & $261 \pm 3$ & $38.5 \pm 0.0$ & $6.3 \pm 0.2$ & $7.38 \pm 0.01$ & $103 \pm 1$ & $34 \pm 1$ \\
\hline $\mathrm{I} / \mathrm{R}+\mathrm{MPG}$ & $90 \pm 1$ & $9 \pm 0$ & $259 \pm 3$ & $38.4 \pm 0.1$ & $6.4 \pm 0.2$ & $7.37 \pm 0.02$ & $103 \pm 1$ & $36 \pm 1$ \\
\hline PostC $+\mathrm{MPG}$ & $89 \pm 1$ & $9 \pm 0$ & $263 \pm 6$ & $38.4 \pm 0.1$ & $6.5 \pm 0.2$ & $7.37 \pm 0.04$ & $108 \pm 1$ & $35 \pm 1$ \\
\hline $\mathrm{I} / \mathrm{R}+(\mathrm{D}) \mathrm{MPG}$ & $87 \pm 2$ & $10 \pm 0$ & $247 \pm 4$ & $38.4 \pm 0.1$ & $6.4 \pm 0.2$ & $7.36 \pm 0.02$ & $105 \pm 1$ & $36 \pm 1$ \\
\hline PostC + (D)MPG & $88 \pm 1$ & $10 \pm 0$ & $266 \pm 4$ & $38.5 \pm 0.0$ & $6.4 \pm 0.1$ & $7.36 \pm 0.02$ & $104 \pm 1$ & $34 \pm 1$ \\
\hline \multicolumn{9}{|l|}{ Reperfusion $10 \mathrm{~min}$} \\
\hline $\mathrm{I} / \mathrm{R}$ & $81 \pm 1$ & $81 \pm 1$ & $261 \pm 5$ & $38.4 \pm 0.1$ & $6.3 \pm 0.2$ & $7.36 \pm 0.02$ & $103 \pm 1$ & $36 \pm 1$ \\
\hline PostC & $79 \pm 1$ & $80 \pm 1$ & $263 \pm 6$ & $38.5 \pm 0.1$ & $6.1 \pm 0.1$ & $7.39 \pm 0.05$ & $104 \pm 1$ & $36 \pm 1$ \\
\hline $\mathrm{I} / \mathrm{R}+\mathrm{MPG}$ & $82 \pm 1$ & $81 \pm 1$ & $260 \pm 5$ & $38.6 \pm 0.1$ & $6.1 \pm 0.1$ & $7.38 \pm 0.04$ & $107 \pm 1$ & $36 \pm 1$ \\
\hline PostC + MPG & $80 \pm 1$ & $82 \pm 1$ & $260 \pm 6$ & $38.5 \pm 0.1$ & $6.3 \pm 0.2$ & $7.39 \pm 0.02$ & $106 \pm 1$ & $36 \pm 1$ \\
\hline $\mathrm{I} / \mathrm{R}+(\mathrm{D}) \mathrm{MPG}$ & $82 \pm 2$ & $80 \pm 1$ & $246 \pm 3$ & $38.5 \pm 0.0$ & $6.5 \pm 0.2$ & $7.36 \pm 0.02$ & $105 \pm 1$ & $36 \pm 1$ \\
\hline PostC + (D)MPG & $82 \pm 1$ & $82 \pm 1$ & $261 \pm 5$ & $38.5 \pm 0.0$ & $6.3 \pm 0.2$ & $7.37 \pm 0.03$ & $103 \pm 1$ & $35 \pm 0$ \\
\hline
\end{tabular}



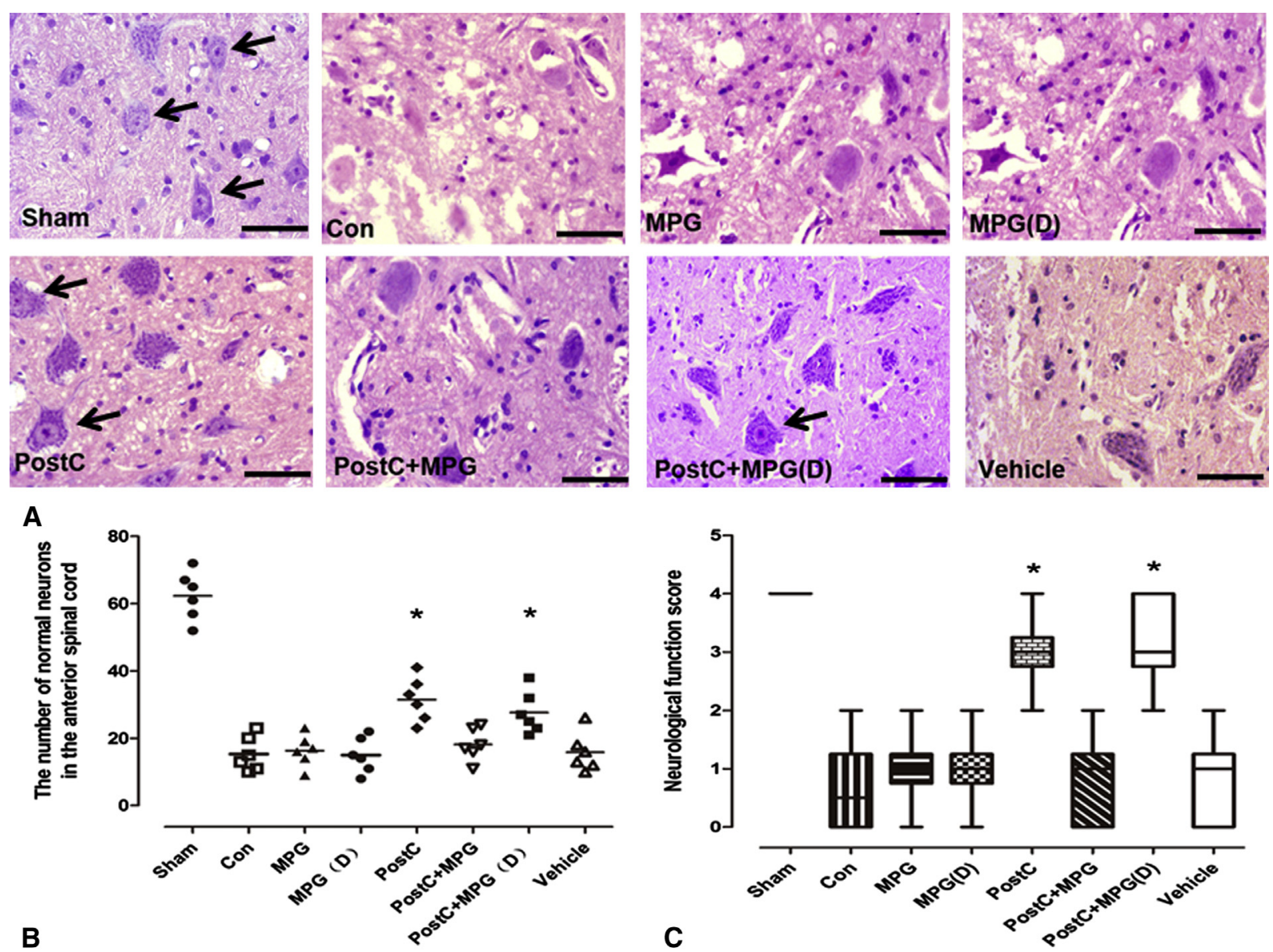

FIGURE 2. Histopathologic results and neurologic function scores 48 hours after reperfusion in each group. A, Representative photomicrographs of lumbar spinal cord sections (L5) in the ventral horn of gray matter 48 hours after reperfusion in 8 groups with hematoxylin-eosin staining. Black arrows indicate Nissl-stained large cells. B, Statistical analysis and comparison of the numbers of normal neurons in the anterior spinal cord among groups $(* P<.001$ vs Con). Each symbol represents data for 1 animal (bar $=$ median). C, Neurologic function scores 48 hours after reperfusion in part 1 ( $\mathrm{n}=6$ in each group). The PostC group showed significantly higher hind-limb motor function scores than the Con group $(P=.002$ vs Con $)$. Nevertheless, the neurologic outcome in the PostC + MPG group was worse than in the PostC group 48 hours after reperfusion. There was no significant difference between the PostC group and the PostC + MPG(D) group $(P=.699)$. Con: Spinal cord underwent 20 minutes of ischemia and 48 hours of reperfusion. Ischemic PostC: Animals received 3 cycles of 30 seconds of reperfusion and 30 seconds of occlusion during the onset of reperfusion. MPG, PostC + MPG: Animals were treated with MPG $\left(2 \%[\mathrm{w} / \mathrm{v}], 0.5 \mathrm{~mL} \cdot \mathrm{kg}^{-1} \cdot \mathrm{min}^{-1}\right.$, intravenously) 10 minutes before reperfusion for 13 minutes. MPG(D) and PostC + MPG(D): Rabbits were treated with delayed MPG 10 minutes after reperfusion for 13 minutes. $M P G$, N-2-mercaptopropionylglycine; PostC, postconditioning.

and catalase $(P<.001$ vs Con $)$ was detected in the spinal cord in the PostC group compared with the Con group 6 hours after spinal cord I/R. Nevertheless, no significant difference was observed between the PostC + MPG and Con groups (SOD $P=.425$ vs Con; catalase $P=.072$ vs Con). In addition, there was no significant difference observed in the 2 antioxidase activities of the spinal cord among the Con, MPG, and PostC + MPG groups (Figure 4, $B$ and $C$ ).

\section{Gene Expressions of $\mathrm{Cu}, \mathrm{Zn}$-SOD and Catalase}

The mRNA expressions of $\mathrm{Cu}, \mathrm{Zn}-\mathrm{SOD}$ and catalase in the spinal cord were also measured. Expression of $\mathrm{Cu}, \mathrm{Zn}$ SOD and catalase mRNA in the sham group was significantly higher than in the other 4 groups (Figure 4).
$\mathrm{Cu}, \mathrm{Zn}$-SOD mRNA expression was notably higher in the PostC group than in the Con group $(P=.005$ vs Con, Figure $4, D$ ), indicating that PostC increased the level of $\mathrm{Cu}, \mathrm{Zn}$-SOD mRNA expression. However, no significant difference was observed between the PostC + MPG and Con groups $(P=.365$ vs Con, Figure $4, D)$, demonstrating that MPG reversed the effect of PostC on Cu,Zn-SOD mRNA expression. Meanwhile, there was no considerable difference in catalase mRNA expression among the Con, MPG, PostC, and PostC + MPG groups (Figure 4, E).

\section{DISCUSSION}

The current study found that ischemic PostC induced a neuroprotective effect in injured spinal cord, accompanied 


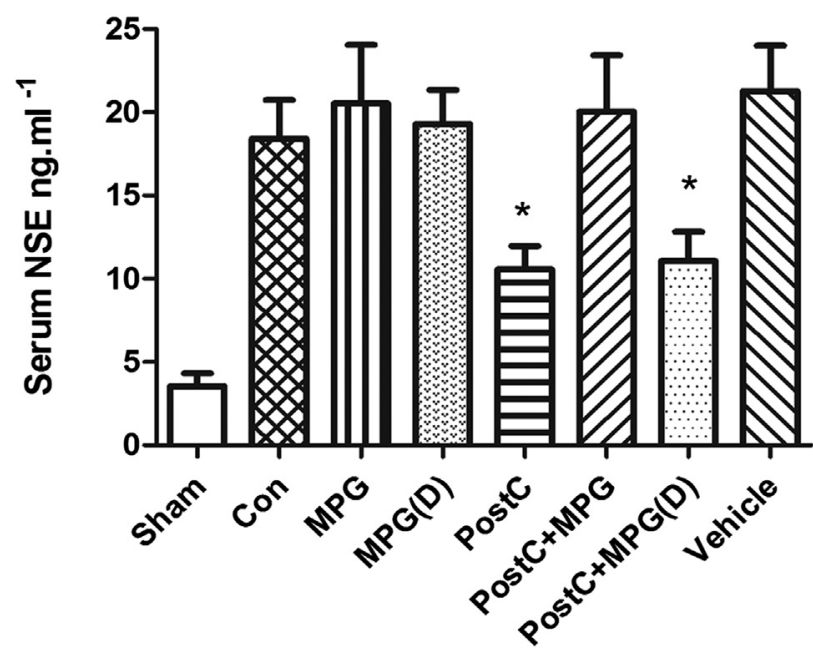

FIGURE 3. Serum NSE levels 48 hours after reperfusion in each group. There was a significant increase in serum NSE levels in the Con group compared with the sham group. NSE levels obtained in PostC $(* P<.05$ vs Con) and PostC $+\operatorname{MPG}(\mathrm{D})\left({ }^{*} P<.05\right.$ vs Con $)$ groups were significantly lower than in the Con group but not than in the sham group. There was no difference in serum NSE levels 48 hours after reperfusion in the PostC and PostC $+\operatorname{MPG}(\mathrm{D})(P=.885)$ groups. NSE, Neuron-specific enolase; $M P G$, N-2-mercaptopropionylglycine; PostC, postconditioning.

by an increase in activities of the major antioxidant enzymes SOD and catalase, and gene expression of $\mathrm{Cu}, \mathrm{Zn}$ SOD in spinal cord tissue, as well as a decrease of the serum biomarker NSE. Moreover, the protective effect and most of the biochemical alteration are attenuated if ROS is eliminated by its scavenger MPG administered 10 minutes before reperfusion, indicating that an antioxidant mechanism is associated with the mechanism of production of neuroprotection of ischemic PostC.

Our previous experiments demonstrated that implementation of three 30/30-second cycles of ischemic PostC at the onset of reperfusion in a rabbit model with 20 minutes of ischemia reduced spinal cord injury. ${ }^{7}$ However, the cellular mechanism that leads to the induction of spinal cord protection is not fully understood.

The results from the present study indicate that the mechanism underlying the protective effect involves intracellular redox signaling. First, we measured the activity of endogenous antioxidant enzymes. The results showed that PostC induced an increase in the activities of the major antioxidant enzymes SOD and catalase in tissue. Although it is well known that a burst of ROS contributes to reperfusion injury, the ROS scavenger MPG administered before reperfusion restrains the spinal cord protection of ischemic PostC, demonstrating that the generation of ROS by PostC is critical for its effect. Antioxidant enzyme activities are highly associated with the ROS generation during PostC (particularly during early reperfusion). Next, we demonstrated that a PostC-induced appropriate increase of ROS upregulates the endogenous antioxidant enzyme activities. The results suggest that redox signaling involves a mechanism of a neuroprotective effect induced by PostC against spinal cord ischemia.

As one of the pivotal enzymes in the antioxidant enzyme system of the body, SOD plays a key part in the body's response to oxidative stress damage. There are 2 types of SOD: $\mathrm{Cu}, \mathrm{Zn}-\mathrm{SOD}$ and $\mathrm{Mn}-\mathrm{SOD}$, and $\mathrm{Cu}, \mathrm{Zn}-\mathrm{SOD}$ forms $80 \%$ of total SOD. ${ }^{18}$ Therefore, we measured the mRNA expression of $\mathrm{Cu}, \mathrm{Zn}-\mathrm{SOD}$ and another antioxidant enzyme, catalase. The results showed that PostC induced an increase in the expression of $\mathrm{Cu}, \mathrm{Zn}-\mathrm{SOD} \mathrm{mRNA}$ in tissue, and this change could be reversed by MPG (a ROS scavenger) given before reperfusion, indicating that PostC increased the generation of ROS and in turn stimulated the expression of $\mathrm{Cu}, \mathrm{Zn}-\mathrm{SOD}$ mRNA. However, in the present study, although PostC increased the activity of catalase, no alteration was found in its mRNA expression in the PostC group compared with the Con group. This was consistent with several other studies. Ciqremis ${ }^{18}$ and colleagues found that oxidative stress in the small intestine of diabetic rats resulted in elevated catalase and SOD activities, whereas their mRNA expressions were unchanged. We speculated that PostC induced a neuroprotective effect mainly through SOD upregulation to reduce oxidative stress injury after reperfusion.

During ischemia, a burst of ROS production may lead to tissue damage and even subsequent cell death. ${ }^{8}$ However, the function of ROS in the protective pathway is more subtle during the reperfusion phase. The current study found that MPG inhibited the neuroprotective effect against spinal cord ischemia if given before PostC, indicating that ROS production during early reperfusion is an important step toward limiting I/R injury. This is in line with previous studies demonstrating that ROS release can be protective. ${ }^{20}$ This is also in line with the observation that $\mathrm{N}$-acetyl cysteine (a type of ROS scavenger) administered during the initial 3 minutes of reperfusion, but not delayed $\mathrm{N}$-acetyl cysteine, altered the protective effect of PostC against heart ischemia. $^{21}$

In the clinic, spinal cord ischemia may be one complication of aortic reconstructive procedures or spinal cord surgery for tumor or vascular malformation. If spinal cord ischemia (eg, occlusion of aorta or hypoperfusion) occurred during the surgical procedure, controlled reperfusion (PostC) might be used before to reduce final reperfusion injury. However, the safety and effectiveness of the clinical application of PostC in patients require further study.

The results from the present study are in agreement with our earlier observation, which showed that spinal cord protection induced by sevoflurane PostC was via ROS-mediated SOD and catalase activity. ${ }^{17}$ Likewise, Liu $^{8}$ showed that PostC protected CA1 neuronal injury and increased SOD and catalase activity in a brain I/R model. 

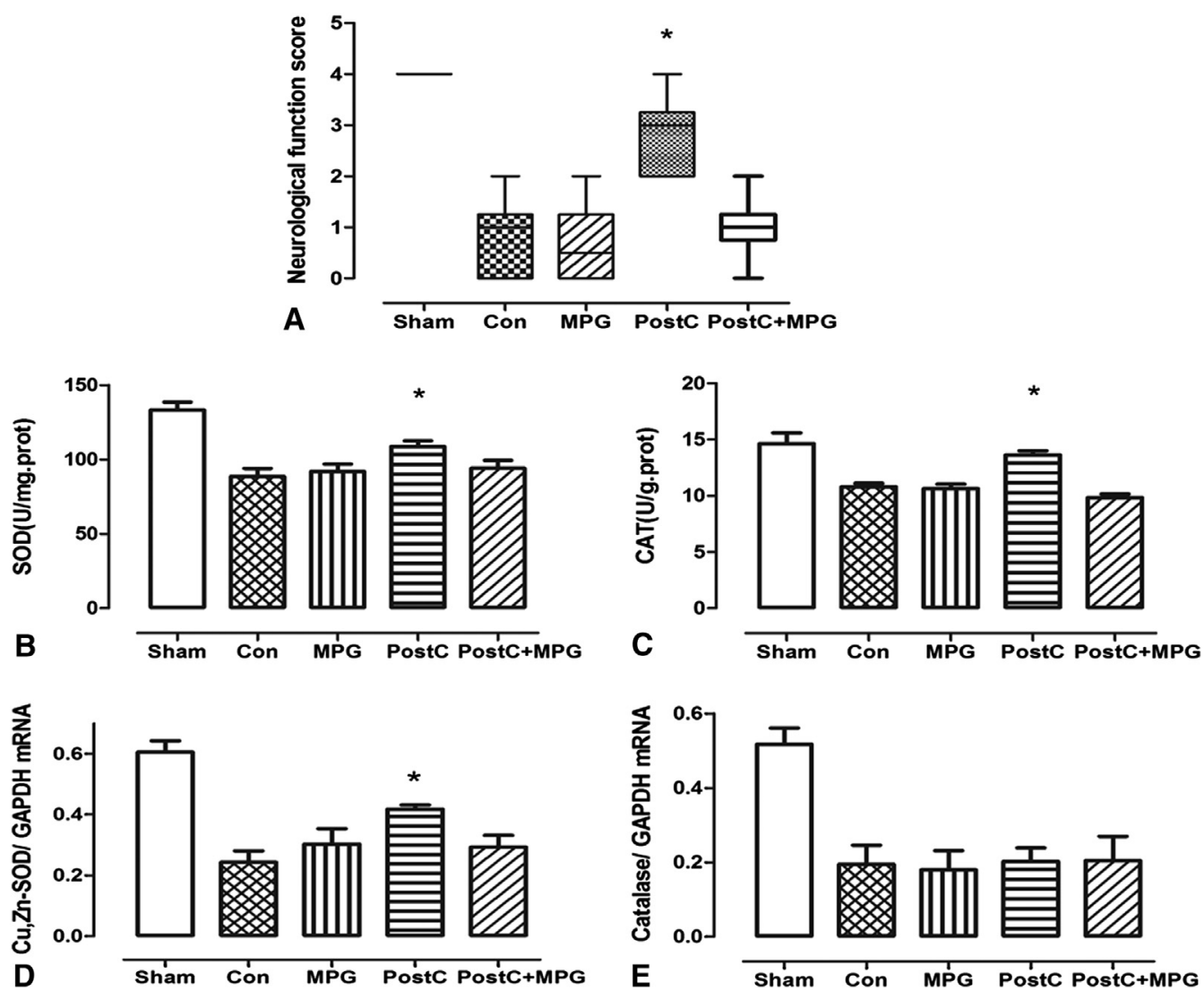

FIGURE 4. Neurologic function scores (A), effect of MPG on SOD (B) and catalase (C) activities ( $\mathrm{n}=6$ for each group), and $\mathrm{mRNA}$ expression of Cu,ZnSOD and catalase ( $\mathrm{n}=4$ for each group) in the spinal cord tissue 6 hours after reperfusion. A, Neurologic function scores in part 2 . Data are expressed as the median (range). $* P<.05$ versus Con group. B, Higher SOD activity was found in the spinal cord in the PostC group compared with the other 3 groups $\left({ }^{*} P<.05\right.$ vs Con). SOD activities in the spinal cord in the PostC + MPG group showed no difference compared with the Con group $(P=.425$ vs Con). $\mathrm{C}$, Higher catalase activity was found in the spinal cord in the PostC group compared with the Con group $(* P<.05$ vs Con). D, Higher mRNA level of $\mathrm{Cu}, \mathrm{Zn}-\mathrm{SOD}$ was found in the spinal cord in the PostC group compared with the Con group $\left({ }^{*} P<.05\right.$ vs Con). E, The mRNA level of catalase in each group. Con: Spinal cord underwent 20 minutes of ischemia and 48 hours of reperfusion. Ischemic PostC group received 3 cycles of 30 seconds of reperfusion and 30 seconds of occlusion during the onset of reperfusion. MPG: the potent ROS scavenger MPG $\left(2 \%[\mathrm{w} / \mathrm{v}], 0.5 \mathrm{~mL} \cdot \mathrm{kg}^{-1} \cdot \mathrm{min}^{-1}\right)$ was administered intravenously 10 minutes before reperfusion for 13 minutes. GAPDH, Glyceraldehyde 3-phosphate dehydrogenase; SOD, superoxide dismutase; $M P G$, N-2mercaptopropionylglycine; PostC, postconditioning; $m R N A$, messenger RNA; CAT, catalase.

\section{Study Limitations}

The current study has some limitations. Previous studies have demonstrated that cardiomyocyte protection induced by PostC is associated with ROS release from mitochondria after opening of the $\mathrm{mK}_{\mathrm{ATP}}$ channel. ${ }^{20,22}$ Whether the $\mathrm{mK}_{\mathrm{ATP}}$ channel and other mediators or effectors mediating the protective effect of PostC in myocytes could also participate in the induction of ischemic tolerance by PostC in the spinal cord remains to be clarified.

\section{CONCLUSIONS}

Overall, our data support the idea that PostC can protect neurons from injury by upregulating the endogenous antioxidants SOD and catalase. This effect can be blockaded by a ROS scavenger administered at an early stage of PostC. Additional investigation is required to explore the precise role of ROS and its pathways in ischemic PostC after injury.

\section{References}

1. Cambria RP, Clouse WD, Davison JK, Dunn PF, Corey M, Dorer D. Thoracoabdominal aneurysm repair: results with 337 operations performed over a 15-year interval. Ann Surg. 2002;236:471-9.

2. Coselli JS, LeMaire SA, Miller CR, Schmittling ZC, Koksoy C, Pagan J, et al. Mortality and paraplegia after thoracoabdominal aortic aneurysm repair: a risk factor analysis. Ann Thorac Surg. 2000;69:409-14.

3. Follette DM, Fey K, Buckberg GD, Helly JJ, Steed DL, Foglia RP, et al. Reducing postischemic damage by temporary modification of reperfusate calcium, potassium, pH, and osmolarity. J Thorac Cardiovasc Surg. 1981;82:221-38.

4. Murry CE, Jennings RB, Reimer KA. Preconditioning with ischemia: a delay of lethal cell injury in ischemic myocardium. Circulation. 1986;74:1124-36.

5. Zhao ZQ, Corvera JS, Halkos ME, Kerendi F, Wang NP, Guyton RA, et al. Inhibition of myocardial injury by ischemic postconditioning during reperfusion: comparison with ischemic preconditioning. Am J Physiol Heart Circ Physiol. 2003;285:579-88. 
6. Staat P, Rioufol G, Piot C, Cottin Y, Cung TT, L'Huillier I, et al. Postconditioning the human heart. Circulation. 2005;112:2143-8.

7. Song WY, Dong HL, Cheng Q, Lu ZH, Wang H, Meng JR, et al. Ischemic postconditioning induces neuroprotection via up-regulation of endogenous antioxidant enzyme activities: experiment with rabbits. Zhonghua Yi Xue Za Zhi. 2008;88:2355-9.

8. Liu PK. Ischemia-reperfusion-related repair deficit after oxidative stress: implications of faulty transcripts in neuronal sensitivity after brain injury. J Biomed Sci. 2003;10:4-13.

9. Nie H, Xiong L, Lao N, Chen S, Xu N, Zhu Z. Hyperbaric oxygen preconditioning induces tolerance against spinal cord ischemia by upregulation of antioxidant enzymes in rabbits. J Cereb Blood Flow Metab. 2006;26:666-74.

10. Tsutsumi YM, Yokoyama T, Horikawa Y, Roth DM, Patel HH. Reactive oxygen species trigger ischemic and pharmacological postconditioning: in vivo and in vitro characterization. Life Sci. 2007;81:1223-7.

11. Penna C, Mancardi D, Rastaldo R, Pagliaro P. Cardioprotection: a radical view Free radicals in pre and postconditioning. Biochim Biophys Acta. 2009;1787: 781-93.

12. Cohen MV, Yang XM, Downey JM. Acidosis, oxygen, and interference with mitochondrial permeability transition pore formation in the early minutes of reperfusion are critical to postconditioning's success. Basic Res Cardiol. 2008;103: 464-71.

13. Wang Q, Ding Q, Zhou Y, Gou X, Hou L, Chen S, et al. Ethyl pyruvate attenuates spinal cord ischemic injury with a wide therapeutic window through inhibiting high-mobility group box 1 release in rabbits. Anesthesiology. 2009; 110:1279-86

14. Shi E, Jiang X, Kazui T, Washiyama N, Yamashita K, Terada H, et al. Nonviral gene transfer of hepatocyte growth factor attenuates neurologic injury after spinal cord ischemia in rabbits. J Thorac Cardiovasc Surg. 2006;132:941-7.
15. Sirlak M, Eryilmaz S, Bahadir Inan M, Sirin YS, Besalti O, Yazicioglu L, et al Effects of carbamazepine on spinal cord ischemia. J Thorac Cardiovasc Surg. 2008;136:1038-43.

16. Dong HL, Zhang Y, Su BX, Zhu ZH, Gu QH, Sang HF, et al. Limb remote ischemic preconditioning protects the spinal cord from ischemia-reperfusion injury: a newly identified nonneuronal but reactive oxygen species-dependent pathway. Anesthesiology. 2010;112:881-91.

17. Wang Q, Chen Q, Ding Q, Yang Q, Peng Y, Lu Y, et al. Sevoflurane postconditioning attenuates spinal cord reperfusion injury through free radicalsmediated up-regulation of antioxidant enzymes in rabbits. J Surg Res. 2011; 169:292-300

18. Ciqremis Y, Turel H, Adiquzel K, Akqoz M, Kart A, Karaman M, et al. The effects of acute acetaminophen toxicity on hepatic Mrna expression of SOD, CAT, GSH-Px, and levels of peroxynitrite, nitric oxide, reduced glutathione, and malondialdehyde in rabbit. Mol Cell Biochem. 2009;323:31-8.

19. Su B, Dong H, Ma R, Zhang X, Ding Q, Xiong L. Cannabinoid 1 receptor mediation of spinal cord ischemic tolerance induced by limb remote ischemia preconditioning in rats. J Thorac Cardiovasc Surg. 2009;138:1409-16.

20. Penna C, Rastaldo R, Mancardi D, Raimondo S, Cappello S, Gattullo D, et al Post-conditioning induced cardioprotection requires signaling through a redoxsensitive mechanism, mitochondrial ATP-sensitive $\mathrm{K}+$ channel and protein kinase C activation. Basic Res Cardiol. 2006;101:180-9.

21. Rastaldo R, Cappello S, Folino A, Di Stilo A, Chegaev K, Tritto I, et al. Low concentrations of an nitric oxide-donor combined with a liposoluble antioxidan compound enhance protection against reperfusion injury in isolated rat hearts. J Physiol Pharmacol. 2010;61:21-7.

22. Penna C, Mancardi D, Rastaldo R, Losano G, Pagliaro P. Intermittent activation of bradykinin B2 receptors and mitochondrial KATP channels trigger cardiac postconditioning through redox signaling. Cardiovasc Res. 2007;75:168-77. 